\title{
Manipulação e visualização de superfícies quádricas por meio de modelos impressos em 3D e modelos digitais
}

Érika Maria Chioca Lopes - UFU - erikalopes@ufu.br - https://orcid.org/0000-0003-3817-5511 Edson Agustini - UFU - agustini@ufu.br - https://orcid.org/0000-0001-8301-865X

Rosana Sueli da Motta Jafelice - UFU - rmotta@ufu.br - https://orcid.org/0000-0001-8489-3974 Arlindo José de Souza Junior - UFU - arlindo@ufu.br - https://orcid.org/0000-0002-5175-6129

\section{Resumo}

Neste artigo, apresentam-se resultados de uma pesquisa aplicada cujos dados empíricos foram obtidos de uma experiência pedagógica usando tecnologias digitais com 17 estudantes do Ensino Superior. O objetivo é compreender as possibilidades que a atividade experimental, desenvolvida com a utilização de modelos de superfícies quádricas produzidos em impressora $3 \mathrm{D}$, trazem ao processo de ensinar e aprender Geometria Analítica. Os dados da pesquisa advieram de entrevistas concedidas pelos participantes da experiência, além de consulta aos arquivos e documentos da proposta. $\mathrm{Na}$ atividade didática, os estudantes se envolveram na manipulação, visualização e interpretação sobre formatos, medidas, posições e propriedades das superfícies. Os resultados permitiram a elaboração de uma proposta baseada em experimentações em um applet no GeoGebra, que possibilita integrar as explorações em modelos digitais de superfícies quádricas ao trabalho com modelos impressos em 3D.

Palavras-chave: impressora 3D; geogebra; superfícies quádricas; geometria analítica.

\section{Manipulation and visualization of quadric surfaces using 3D printed models and digital models}

\begin{abstract}
In this paper, one presents results of an applied research whose empirical data was obtained from a pedagogical experience using digital technologies with 17 students of Higher Education. The aim is to understand the possibilities that the experimental activity, developed with the use of quadric surfaces models produced in a 3D printer, brings to the process of teaching and learning Analytic Geometry. The research data came from interviews given by the participants of the experiment, in addition to consulting the files and documents of the proposal. In the didactic activity, students were involved in the manipulation, visualization and interpretation of formats, measures, positions and properties of surfaces. The results allow one to elaborate a proposal based on experiments in an applet in GeoGebra, which makes it possible to integrate explorations in digital models of quadric surfaces to work with 3D printed models.
\end{abstract}

Keywords: 3D printer; geogebra; quadric surfaces; analytic geometry.

\section{Introdução}

O ensino de Geometria Analítica (GA) na universidade, juntamente com o de Cálculo Diferencial e Integral, tem sido discutido por docentes, coordenadores e pesquisadores há muito tempo. Por serem disciplinas básicas, que introduzem os ingressantes ao estudo de conteúdos matemáticos necessários para a continuidade de sua formação universitária, a aprendizagem deficiente e o baixo desempenho têm graves consequências (DI PINTO, 2000; RICHIT, 2005).

Com a intenção de se enfrentar o problema das altas taxas de retenção e evasão em determinadas disciplinas, foi apresentado à comunidade acadêmica da Universidade V. $19 \mathrm{~N}^{\circ} 1$, julho, 2021

DOI: https://doi.org/10.22456/1679-1916.118529 RENOTE 
Federal de Uberlândia o Programa Institucional de Graduação Assistida (PROSSIGA). A coordenação do programa abriu edital para projetos que buscassem promover ações voltadas para o aprimoramento do aprendizado acadêmico; desenvolver atividades, métodos e práticas pedagógicas inovadoras; e ampliar oportunidades de estudos pelos estudantes de graduação.

Com esse objetivo, formou-se um grupo de trabalho na Faculdade de Matemática para elaboração de um projeto voltado para o ensino e a aprendizagem de GA com tecnologias digitais, denominado PROSSIGA-GA. Esse projeto incluiu a ação conjunta de professores e estudantes bolsistas no ensino presencial de GA em diversos Cursos de Graduação, em dois semestres letivos, complementada por um ambiente de aprendizagem na plataforma Moodle, a utilização do software GeoGebra e de modelos de superfícies quádricas, produzidos por meio de uma impressora 3D.

Neste artigo, busca-se compreender possibilidades que a prática pedagógica desenvolvida com a utilização de modelos de superfícies quádricas, produzidos em impressora 3D, trazem para o processo de ensinar e aprender GA. Trata-se de um recorte de uma pesquisa aplicada (LOPES, 2019), de abordagem qualitativa, a qual procurou analisar diversos aspectos desse processo, por meio de dados empíricos obtidos nessa experiência.

Para a análise dos dados produzidos nesta pesquisa, apoiou-se na Epistemologia Qualitativa de González Rey (2005), cujos princípios podem ser assim resumidos: a) consideração do conhecimento como construção/produção humana; b) consideração dos registros empíricos como parte da produção teórica da pesquisa qualitativa; e c) compreensão da pesquisa nas ciências antropossociais como um processo dialógico.

Nessa perspectiva, tomaram-se como fontes de dados os documentos disponíveis (projeto inicial, relatórios, planos de ensino) e as entrevistas semiestruturadas concedidas por 17 estudantes, que cursaram a disciplina de GA durante a implementação dessa proposta pedagógica. A partir da leitura das transcrições das entrevistas, entrelaçadas com as reflexões produzidas a partir da literatura científica, realizou-se o processo analítico de codificação, categorização e sínteses. (DUARTE, 2004).

\section{Problemática acerca do ensino e da aprendizagem de Geometria Analítica}

Em Geometria Analítica no Ensino Superior, os estudantes devem aprender a representar objetos geométricos bi ou tridimensionais - retas, planos, curvas, superfícies - por meio de equações variadas e, a partir desse estudo, concluir propriedades e características de tais objetos. Inicialmente, parte-se da utilização de um sistema de coordenadas para estabelecer uma correspondência entre pares (ou trios) ordenados de números reais e pontos do plano (ou do espaço). Dessa forma, relaciona-se geometria e álgebra e busca-se resolver problemas fazendo uso conveniente das duas representações.

Vários pesquisadores salientam a necessidade de desenvolvimento do pensamento visual, predominante na geometria, bem como o pensamento algébrico (ATIYAH, 1982, PAVANELLO, 1993). Quanto à visualização, Flores (2003) argumenta sobre como imagens podem ser utilizadas para elaborar e transmitir saberes, donde decorre a importância de se incentivar essa habilidade nos meios educacionais.

Por outro lado, Giardinetto (1991) discute como a dicotomia entre o concreto e o abstrato se manifesta no ensino de GA na Educação Básica, o qual se reduz a uma associação mecânica entre o grau da equação algébrica e sua correspondente curva, sem apreensão de significado. Dessa forma, o estudo das retas e curvas cônicas é abordado apenas do ponto de vista algébrico. Também Richit (2005, p. 21) argumenta que a forma como a disciplina de Geometria Analítica tem sido tratada, tanto no Ensino 
Médio quanto Superior, agrava o problema das altas reprovações, “[...] à medida que há uma lacuna na transição da representação geométrica para algébrica de conceitos e propriedades inerentes à mesma.".

No estudo de superfícies quádricas, um dos tópicos do programa de GA nas universidades, também nota-se que o estudante utiliza a memorização para fazer a associação entre o grau e o sinal das variáveis cartesianas na equação e a correspondente classificação da superfície. Em geral, também percebem-se dificuldades para identificação da representação geométrica da superfície.

Com os avanços tecnológicos nas últimas décadas, muitas pesquisas apontam que a utilização de alguns softwares, especialmente os de geometria dinâmica, têm favorecido a visualização e a manipulação de figuras e enriquecido a aprendizagem (Gravina, 2015). Mas, muito antes disso, pesquisadores analisavam as potencialidades da utilização de materiais físicos e manipuláveis para o ensino de Matemática. Fiorentini e Miorim (1990, p.5) destacaram:

\begin{abstract}
Ao aluno deve ser dado o direito de aprender. Não um "aprender" mecânico, repetitivo, de fazer sem saber o que faz e porque faz. Muito menos um "aprender" que se esvazia em brincadeiras. Mas um aprender significativo, do qual o aluno participe raciocinando, compreendendo, reelaborando o saber historicamente produzido e superando, assim, sua visão ingênua, fragmentada e parcial da realidade. $\mathrm{O}$ material ou o jogo pode ser fundamental para que isso ocorra.
\end{abstract}

LOPES (2019) registrou duas experiências que utilizaram materiais físicos em propostas com superfícies quádricas em GA na universidade. ${ }^{1}$

$\mathrm{Na}$ pesquisa de Mineiro (2011), alguns modelos de representação tridimensional foram utilizados em uma intervenção com estudantes do $3^{\circ}$ ano de um curso de Licenciatura em Matemática. Para a criação dos modelos, foram impressos gráficos bidimensionais em folhas de acetato transparente, correspondentes às curvas de nível de uma determinada superfície quádrica. As folhas transparentes foram perfuradas e coladas, a distâncias iguais, em hastes formadas por canudos plásticos. No desenvolvimento da pesquisa, foram propostas várias atividades, entre as quais, era solicitado aos estudantes, divididos em grupos, que fizessem a montagem livre de uma superfície, por meio da colagem das folhas já impressas com circunferências. A partir dos protocolos das atividades, eles debatiam sobre as questões e as respondiam.

Vasconcelos et al. (2010) apresentam um método de construção de modelos concretos, que representam sólidos e superfícies que são boas aproximações físicas dos objetos geométricos, desenvolvido no Laboratório de Ensino de Matemática da UFBA (LEMA-UFBA). Para a confecção dessas superfícies, uma artista plástica que atuava no laboratório utilizou papel, cola, massa acrílica e tinta, que eram aplicados a moldes feitos com uso de recursos computacionais. O método de construção dos moldes concretos era baseado em interseções da superfície com um conjunto de planos paralelos, a partir de aplicativos computacionais. Segundo as autoras, os sólidos e superfícies eram utilizados nas aulas de Cálculo, Geometria Analítica e Geometria Diferencial.

No ensino de superfícies nas disciplinas de GA das universidades, são raras as experiências divulgadas de utilização de objetos tridimensionais físicos e manipuláveis pelos estudantes. O ponto comum a esses dois casos citados é combinar a precisão com o toque artístico. Os cortes das superfícies foram modelados a partir de equações com o auxílio computacional, enquanto que a montagem em ambiente tridimensional foi

\footnotetext{
${ }^{1}$ Uma pesquisa de mestrado, defendida em 2011, e um livro editado na Universidade Federal da Bahia (UFBA), em 2010.

V. $19 \mathrm{~N}^{\mathrm{o}} 1$, julho, 2021

DOI: https://doi.org/10.22456/1679-1916.118529 RENOTE
} 
artesanal. Já no projeto PROSSIGA-GA, todo o processo de confecção física dos objetos é digital e exato, desde a utilização de equações para a modelagem computacional até sua execução em impressora 3D.

\section{Experiência com modelos impressos em 3D no contexto do PROSSIGA-GA}

Conforme dito, uma das vertentes do trabalho desenvolvido no PROSSIGA-GA foi a produção de superfícies quádricas por um bolsista do projeto, ligado ao Laboratório de Engenharia Biomédica, por meio de impressora 3D disponibilizada nesse local. Essa etapa do projeto ocorreu durante cerca de dez meses, período no qual houve a elaboração dos modelos computacionais ${ }^{2}$ de cada superfície, os ajustes com o grupo de trabalho do PROSSIGA-GA e a impressão dos modelos 3D.

Os modelos produzidos foram utilizados em algumas aulas presenciais, para complementar as discussões teóricas e exposições via GeoGebra sobre esse assunto. A Figura 1 mostra todas as superfícies produzidas durante o projeto: superfície esférica, elipsoide, hiperboloides de uma folha (circular e elíptico), hiperboloides de duas folhas (circular e elíptico), paraboloides (circular e elíptico), paraboloide hiperbólico, superfície cilíndrica circular, superfície cônica circular de duas folhas e um sistema de coordenadas cartesianas ortogonais no espaço .

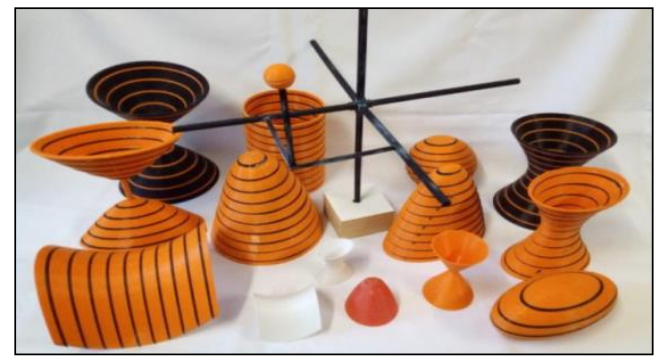

Figura 1 - Modelos de superfícies produzidas em impressora 3D Fonte: arquivos do PROSSIGA-GA

A interatividade dos estudantes com essas superfícies ocorreu durante algumas aulas conduzidas pelo professor, com participação dos mesmos. Nas entrevistas realizadas, muitos estudantes se manifestaram, mostrando interesse e motivação, como os seguintes: "Eu achei interessante ter na realidade... tipo ser palpável o que você está aprendendo. Ver uma equação algébrica e ter nas mãos o quê aquilo significa, aquele conjunto de pontos". e "Eu acho que o mais interessante foi o toque mesmo, de poder pegar, para ver o quê que é, porque até quando é para a gente ver por vídeo, a gente precisa de imaginar.”.

Os comentários dos estudantes permitem identificar a importância que eles colocaram na experiência tátil, associada à percepção visual, que possibilitou que visualizassem detalhes das superfícies a partir da sua manipulação física, detalhes que não seriam tão fáceis de visualizar no GeoGebra ou num vídeo. Isso também foi identificado por Lemke, Siple e Figueiredo (2016, p. 8), em pesquisa que fizeram sobre a utilização de applets do GeoGebra e maquetes físicas produzidas em impressora 3D, para o ensino de Cálculo 2.

Bates (2017) diz que os sentidos utilizados pelos usuários para interpretar as mídias são audição e visão. Nesse caso, identifica-se que o tato pode complementar a experiência, no sentido de ampliar as possibilidades de desenvolvimento da percepção visual dos estudantes. Ao contrário dos modelos digitais de superfícies, que eram 'movimentados' pelo professor no GeoGebra, as superfícies quádricas produzidas nesse

\footnotetext{
${ }^{2}$ Esses programas computacionais eram elaborados no software AUTOCAD, um software comercial de Desenho Assitido por computador (DAC), ou CAD (do inglês: computer aided design), utilizado em geral para criação de projetos em engenharia.
} 
projeto podem ser manipuladas 'com as próprias mãos' pelos estudantes e, ainda, possibilitam que sejam produzidos significados com relação à forma, ao volume e às interseções das superfícies com determinados planos no espaço.

Um fato interessante foi identificado nas entrevistas de dois estudantes, que comentaram sobre a vantagem da manipulação das superfícies impressas para a aprendizagem do conceito de superfície regrada. $\mathrm{Na}$ aula, um dos modelos manipulados por eles foi de um hiperboloide de uma folha. A partir da explicação, realizada pelo professor, do que é uma superfície regrada ${ }^{3}$ (Figura 2), e da manipulação feita pelos estudantes, de deslizar uma régua sobre o modelo, foi possível verificar intuitivamente essa propriedade, que pode ser demonstrada analiticamente. Ficou claro, nas falas dos estudantes, que a experiência vivenciada foi marcante para a consolidação da aprendizagem desse conceito: "O hiperboloide de uma folha...que a gente ia com a régua e ia vendo que todas as geratrizes dele se encaixavam, então, ajudou bastante ver, tocar aquilo.".

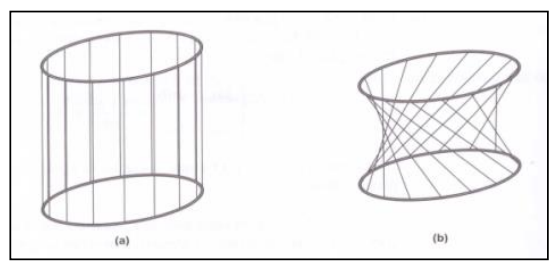

Figura 2 - Hiperboloide de uma folha (fig. b), obtido a partir de um cilindro elíptico (fig. a) Fonte: CAMARGO; BOULOS (2005, p. 409)

Diante disso, alinha-se às conclusões de Hoffmann, Martins e Basso (2009, p. 4), de que "[...] tanto o trabalho com o material digital quanto com o não-digital utilizados em experiências físicas podem servir de base para as experiências lógico-matemáticas".

Outro fato identificado em algumas entrevistas foi a maneira como o professor conduziu a 'conversa' em sala de aula, por meio de perguntas que levavam os estudantes a raciocinar os efeitos sobre a figura de alterações em coeficientes da equação: "O professor cobrava, por exemplo, num hiperboloide, se eu diminuir ele assim, o que acontece? Se eu diminuir ele assim, o que acontece [gesticulando]?”. Os relatos mostram que é possível fazer um trabalho integrado entre as abordagens algébrica e geométrica para o ensino das superfícies, caminho indicado por Giardinetto (1991) para superação da dicotomia entre essas abordagens. Assim, ao questionar o estudante sobre os efeitos algébricos produzidos por mudanças no formato da superfície que ele tinha em mãos, desperta-se a necessidade de entender o significado de cada parâmetro e cada variável que aparece na equação que modela a figura, em termos geométricos.

Além disso, esses comentários evidenciam o papel importante que deve ser desempenhado pelo professor em momentos de utilização de materiais manipuláveis para o ensino de matemática, pois apenas ter os objetos em mãos não significa que o estudante vai construir algum conhecimento. Corrobora-se com Vale (2002), ao apontar que somente as pessoas, com sua mente, podem gerar ou enxergar matemática a partir de materiais manipuláveis. Assim, em contextos educacionais, é preciso que o professor crie situações que possibilitem ao estudante viver experiências com a utilização desses materiais, que o levem ao pensamento matemático.

\section{Possibilidades de integração entre modelos impressos em 3D e modelos digitais}

Se, por um lado, são raras as experiências que utilizam materiais físicos tridimensionais para o ensino de superfícies, por outro, há muitas pesquisas que analisam experiências sobre o ensino e aprendizagem de GA apoiadas em softwares,

\footnotetext{
${ }^{3}$ Uma superfície regrada é uma reunião de retas, embora não possua, e toda a sua extensão, nenhuma região plana, por pequena que seja (CAMARGO; BOULOS, 2005). V. $19 \mathrm{~N}^{\circ} 1$, julho, 2021 DOI: https://doi.org/10.22456/1679-1916.118529 RENOTE
} 
especialmente o GeoGebra, como mostra o mapeamento feito por Souza Junior e Lopes (2017). Entretanto, poucas são voltadas para o tema Superfícies Quádricas.

Nesta seção, como consequência dos resultados obtidos a partir das entrevistas analisadas, apresenta-se uma proposta de integração entre modelos de superfícies produzidos em impressoras 3D e modelos digitais elaborados e visualizados no software GeoGebra. A temática para essa integração será o estudo de equações cartesianas de superfícies quádricas via variação de parâmetros. Para tanto, apresentam-se a seguir algumas breves, mas necessárias, definições para melhor explicar a proposta.

Em disciplinas de Geometria Analítica, é frequentemente apresentada a definição de superfície quádrica:

O lugar geométrico (conjunto) de todos os pontos $P(x, y, z)$ do espaço cartesiano que satisfazem a equação quadrática geral com três variáveis e coeficientes reais

$$
A x^{2}+B y^{2}+C z^{2}+D x y+E x z+F y z+G x+H y+I z+J=0
$$

sendo que algum número real $A, B, C, D, E$ ou $F$ é não nulo (para que a equação seja, de fato, quadrática), é chamado de superfície quádrica.

Geralmente, casos particulares da Equação (1) são considerados, notadamente os casos em que os termos mistos não aparecem, ou seja, $D=E=F=0$, e essa equação pode ser manipulada por meio de completamentos de quadrados e reescrita de forma mais simples no seguinte formato:

$$
s_{1} \frac{\left(x-x_{0}\right)^{p_{1}}}{a^{2}}+s_{2} \frac{\left(y-y_{0}\right)^{p_{2}}}{b^{2}}+s_{3} \frac{\left(z-z_{0}\right)^{p_{3}}}{c^{2}}=k
$$

sendo que $s_{1}, s_{2}, s_{3} \in\{-1,0,1\} ; p_{1}, p_{2}, p_{3} \in\{1,2\} ; k \in\{0,1\} ; a, b, c \in R_{+}$e $x_{0}, y_{0}, z_{0} \in R$, respeitando-se a condição de que ocorra pelo menos um termo quadrático na equação.

Da Equação (2) tiram-se todas as equações reduzidas de superfícies quádricas estudadas nas disciplinas de GA, inclusive as degeneradas.

Chamam-se todas as constantes que aparecem na Equação (2) de parâmetros da equação. Os parâmetros $s_{1}, s_{2}, s_{3}, p_{1}, p_{2}, p_{3}$ e $k$ determinam o tipo de superfície: cilindros, cones, elipsoides, hiperboloides e paraboloides, além de algumas superfícies degeneradas. Já os parâmetros $a, b$ e $c$ estão relacionados com deformações (compressões e dilatações) nas direções dos eixos coordenados, enquanto que os parâmetros $x_{0}, y_{0}$ e $z_{0}$ estão relacionados com deslocamentos (sem deformações) nas direções dos mesmos eixos.

Em termos de aprendizagem, é muito interessante poder ver e comparar duas superfícies quádricas de mesmo tipo no espaço cartesiano (dois elipsoides, por exemplo) e descrever os parâmetros de suas equações. Dizer, por exemplo, se o parâmetro $a$ de um é menor, igual ou maior do que o parâmetro $a$ do outro. Se o estudante souber os efeitos geométricos que os parâmetros impõem sobre as superfícies, as respostas tornam-se fáceis.

Além disso, quando pode-se dispor de diversos modelos de superfícies quádricas, impressos em 3D, de variados tamanhos, comprimidas (achatadas) e dilatadas (esticadas), é relativamente fácil estimular os estudantes a associar a superfície com a equação e comparar seus parâmetros. Isso torna-se mais estimulante ainda com o auxílio de um applet dinâmico no GeoGebra, no qual pode-se fazer as diversas combinações de parâmetros e explorar as deformações e os deslocamentos via controles deslizantes.

Uma proposta para esse applet no GeoGebra está disponibilizada no seguinte endereço eletrônico: https://www.geogebra.org/m/ukztmzxh.

Mais uma vez, cabe ressaltar que o fato do aluno poder manipular um modelo de superfície, impresso em 3D, em suas mãos é um forte diferencial para a aprendizagem e, V. $19 \mathrm{~N}^{\mathrm{o}} 1$, julho, 2021 RENOTE 
felizmente, aproxima-se uma era em que os próprios alunos poderão projetar e imprimir seus modelos geométricos de superfícies em Laboratórios de Ensino de Matemática. Para tanto, cabe mencionar que o próprio software GeoGebra pode ser utilizado para criar arquivos de objetos tridimensionais com extensões STL (STereoLithography) que podem ser lidos e utilizados pelos softwares de fatiamento da maioria das impressoras 3D existentes na atualidade. Neste ponto, ressalta-se que, a depender das características de uma determinada superfície, dois ou mais arquivos STL são necessários para confeccioná-la sem imperfeições. A esfera é um exemplo disso e, até mesmo nesse aspecto, elaborar arquivos STL que resultem em superfícies perfeitas é um ótimo exercício de raciocínio e criatividade ${ }^{4}$.

Também é sempre bom citar que o estímulo ao desenvolvimento de projetos com intercâmbio entre materiais físicos e digitais é citado, inclusive, na BNCC (Base Nacional Comum Curricular) do Ensino Médio brasileiro.

Abaixo apresenta-se, a título de exemplificação, as conclusões do estudo da variação de parâmetros para um elipsoide, feito no applet citado acima. Uma vez entendido o procedimento, o próprio estudante poderá replicar este estudo para as demais superfícies quádricas. As figuras foram tiradas do próprio arquivo.

Tome-se na Equação (2) os seguintes valores de parâmetros: $s_{1}=s_{2}=s_{3}=1$; $p_{1}=p_{2}=p_{3}=2$ e $k=1$. Tem-se, assim, um elipsoide de equação

$$
\frac{\left(x-x_{0}\right)^{2}}{a^{2}}+\frac{\left(y-y_{0}\right)^{2}}{b^{2}}+\frac{\left(z-z_{0}\right)^{2}}{c^{2}}=1 \text {, }
$$

com centro no ponto $C\left(x_{0}, y_{0}, z_{0}\right)$ e eixos paralelos aos eixos coordenados.

Para concluir o que um determinado parâmetro faz, do ponto de vista geométrico, em uma superfície, é sempre bom considerar uma "superfície de referência", que neste caso toma-se como sendo uma esfera com centro na origem e raio 1, colocando $a=b=c=1$ e $x_{0}=y_{0}=z_{0}=0$ na Equação (3). A Figura 3 ilustra as janelas de visualização do arquivo GeoGebra.



Figura 3 - Modelo digital da esfera de centro na origem e raio 1

Fonte: Elaboração dos autores

- Parâmetros $a, b, c$ :

- Para $0<a<1$, tem-se uma compressão da esfera contra o plano $y z$ na direção do eixo $x$. Analogamente para $0<b, c<1$, onde tem-se compressões da esfera contra os planos $x z$ e $x y$ nas direções dos eixos $y$ e $z$, respectivamente. As Figuras 4, 5 e 6 ilustram esses casos.

\footnotetext{
${ }^{4}$ No link a seguir, está uma gravação de parte da produção da esfera, feita na impressora 3D utilizada no PROSSIGA-GA:

https://drive.google.com/file/d/0B_h2IWSgODKzM3hFMkZPXzBScnM/view?usp=sharing\&resourcekey $=0$-9ImSTezfO9CsIIMbM4gQRA

V. $19 \mathrm{~N}^{\mathrm{o}} 1$, julho, 2021

DOI: https://doi.org/10.22456/1679-1916.118529 RENOTE
} 


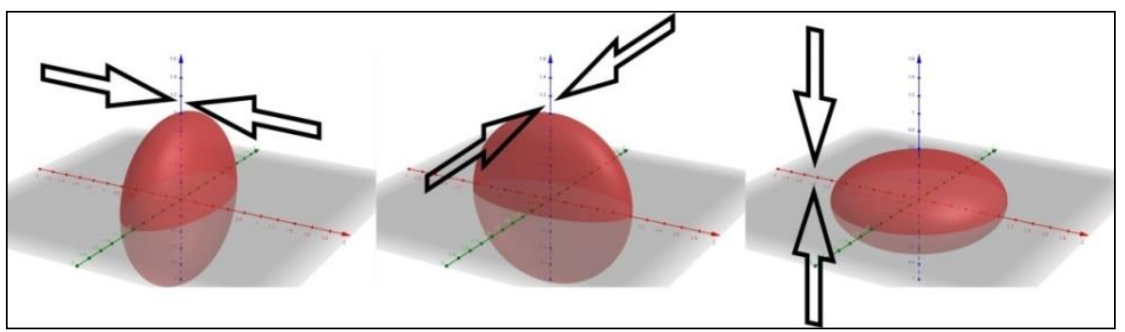

Figuras 4, 5 e 6 - Compressões da esfera para $0<a<1,0<b<1$ e $0<c<1$

Fonte: Elaboração dos autores

- Para $a>1$, tem-se uma dilatação da esfera a partir do plano $y z$ na direção do eixo $x$. Analogamente para $b, c>1$, onde tem-se dilatações da esfera a partir dos planos $x z \mathrm{e}$ $x y$ nas direções dos eixos $y$ e $z$, respectivamente. As Figuras 7, 8 e 9 ilustram esses casos.

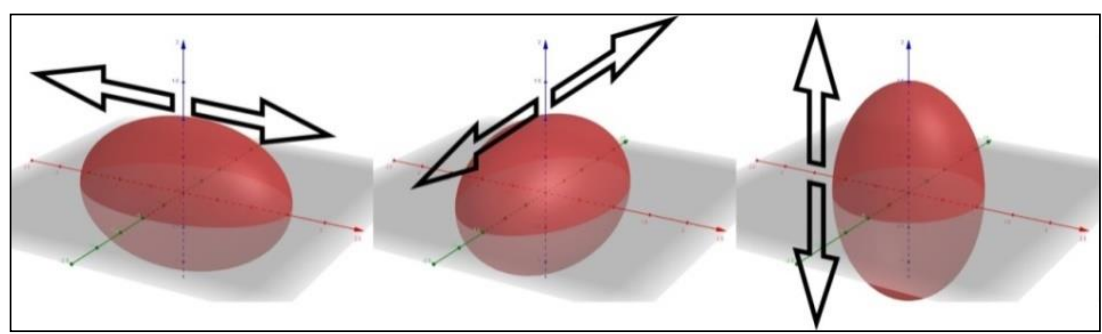

Figuras 7, 8 e 9 - Dilatações da esfera para $a>1, b>1$ e $c>1$

Fonte: Elaboração dos autores

- Parâmetros $x_{0}, y_{0}, z_{0}$ :

- Para $0<x_{0}$, tem-se uma translação de $x_{0}$ unidades do elipsoide ao longo da direção do eixo $x$ no sentido positivo. Analogamente para $0<y_{0}, z_{0}$ nas direções dos eixos $y$ e $z$, respectivamente. As Figuras 10, 11 e 12 ilustram esses casos.

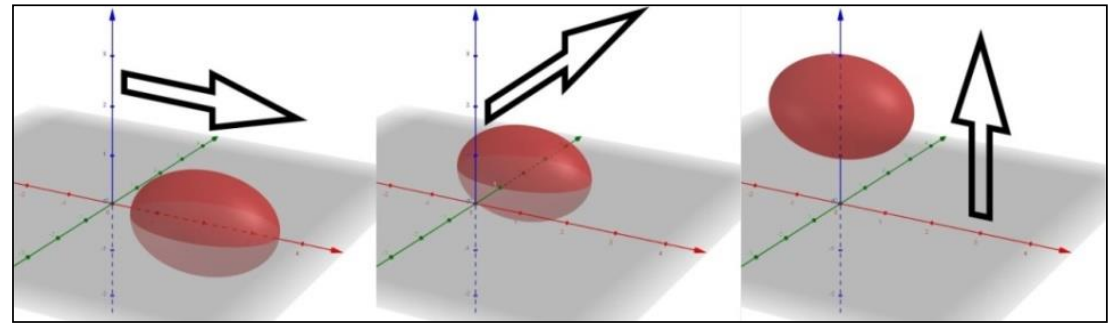

Figuras 10, 11 e 12 - Translações do elipsoide para $0<x_{0}, 0<y_{0}$ e $0<z_{0}$

Fonte: Elaboração dos autores

- Para $x_{0}<0$, tem-se uma translação de $\left|x_{0}\right|$ unidades do elipsoide ao longo da direção do eixo $x$ no sentido negativo. Analogamente para $y_{0}, z_{0}<0$ nas direções dos eixos $y$ e $z$, respectivamente. As Figuras 13, 14 e 15 ilustram esses casos.

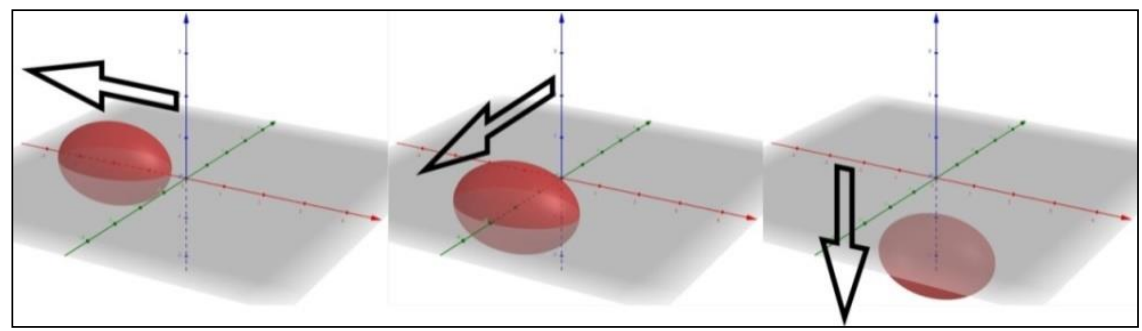

Figuras 13, 14 e 15 - Translações do elipsoide para $x_{0}<0, y_{0}<0$ e $z_{0}<0$

Fonte: Elaboração dos autores 
É claro que pode-se fazer combinações diversas com esses seis parâmetros e observar no applet do GeoGebra que, por exemplo, uma vez alterados os valores de $x_{0}, y_{0}$ e $z_{0}$, as deformações produzidas pelos parâmetros $a, b$ e $c$ ocorrem a partir de planos paralelos aos planos coordenados. Também é natural observar que, por meio de valores adequados para $a, b$ e $c$, pode-se construir elipsoides de qualquer tamanho.

Por fim, ressalta-se que a partir desse estudo, é natural fazer uma extensão de modo a abordar fatiamentos da superfície por planos paralelos aos planos coordenados. Com isso, há uma integração com o estudo de curvas cônicas, que geralmente é feito antes do estudo de superfícies quádricas. Também não pode-se deixar de mencionar que é possível empreender um estudo de ajustes de parâmetros para modelagem em escala reduzida de objetos tridimensionais reais e que podem ser produzidos em impressoras 3D como, por exemplo, maquetes de projetos arquitetônicos.

\section{Considerações Finais}

Buscou-se, neste artigo, compreender as possibilidades que a atividade experimental desenvolvida com a utilização de modelos de superfícies quádricas, produzidos em impressora 3D, trazem para o processo de ensinar e aprender Geometria Analítica. A análise dos dados mostrou que as superfícies produzidas em impressora 3D, manipuladas pelos estudantes, estes conduzidos por questões levantadas pelo professor, favoreceram o entendimento de conceitos e a integração entre as representações algébrica e geométrica das superfícies. A prática educativa desenvolvida com esses modelos em sala de aula mostrou que a experiência tátil foi importante, pois complementou a visualização das figuras geométricas, feita por meio do GeoGebra ou de desenhos no quadro.

Além disso, com foco na relação entre figuras e equações, apresentou-se uma proposta complementar, por meio de um applet dinâmico no GeoGebra. Partindo-se de uma equação cartesiana geral de uma superfície quádrica e sua figura associada, o estudante, sob a orientação do professor, pode realizar experimentações para estudar os efeitos geométricos da variação de cada parâmetro da equação. Alguns recursos existentes hoje no software permitem a exportação de arquivos em formato reconhecido por impressoras 3D. Com isso, abre-se a possibilidade, nessa proposta, de imprimir alguns modelos específicos de superfícies, tornando a discussão sobre a variação de parâmetros 'palpável'. Portanto, abrem-se muitas possibilidades para a realização de trabalhos educativos que integrem modelos digitais de superfícies quádricas, gerados no GeoGebra a modelos impressos em 3D, com a participação ativa dos estudantes.

\section{Referências}

ATIYAH, M. What is geometry? The Mathematical Gazette. v. 66, n. 437, p. 179184, out. 1982.

BATES, A. W. Educar na era digital: design, ensino e aprendizagem. Tradução: João Mattar. São Paulo: Artesanato Educacional, 2017.

CAMARGO, I.; BOULOS, P. Geometria Analítica: um tratamento vetorial. São Paulo: Prentice Hall, 2005. $3^{\text {a }}$ ed. rev. e ampl.

DI PINTO, M. A. Ensino e aprendizagem da Geometria Analítica: as pesquisas brasileiras da década de 90. 2000. Dissertação (Mestrado em Educação Matemática) Pontifícia Universidade Católica de São Paulo, São Paulo, 2000.

DUARTE, Rosália. Entrevistas em pesquisas qualitativas. Educar, Curitiba, n. 24, p. 213-225, 2004. 
FIORENTINI, D; MIORIM, M. A. Uma reflexão sobre o uso de materiais concretos e jogos no ensino da matemática. Boletim SBEM. São Paulo, ano 4, n. 7, jul./ago. 1990.

FLORES, C. R. Olhar, Saber, Representar: ensaios sobre a representação em perspectiva. 2003. Tese (Doutorado em Educação) - Universidade Federal de Santa Catarina, Florianópolis, 2003.

GIARDINETTO, J. R. B. A relação entre o concreto e o abstrato no ensino da geometria analítica a nível do $\mathbf{1}^{\circ}$ e $\mathbf{2}^{\circ}$ Graus. 1991. Dissertação (Mestrado em Educação) - Universidade Federal de São Carlos, São Carlos, 1991.

GONZÁlEZ REY, F. Pesquisa qualitativa e subjetividade: os processos de construção da informação. Tradução: Marcel Aristides Ferrada Silva. São Paulo: Cengage Learning, 2005.

GRAVINA, M. A. O potencial semiótico do GeoGebra na aprendizagem da geometria: uma experiência ilustrativa. Vydia, Santa Maria, v. 35, n. 2, jul. 2015. p. 237-253.

HOFFMANN, D. S.; MARTINS, E. F.; BASSO, M. V. A. Experiências física e lógicomatemática em espaço e forma: uma arquitetura pedagógica de uso integrado de recursos manipulativos digitais e não-digitais. In: SIMPÓSIO BRASILEIRO DE INFORMÁTICA NA EDUCAÇÃO, XX, 2009, Santa Catarina. Anais [...]. Santa Catarina: UFSC, 2009. p. 1-10.

LEMKE, R.; SIPLE, I. Z.; FIGUEIREDO, E. B. OAs para o ensino de cálculo: potencialidades de tecnologias 3D. Revista Novas Tecnologias na Educação, Porto Alegre, v. 14, n. 1, p. 1-10, jul. 2016.

LOPES, E. M. C. Integração de mídias na disciplina de geometria analítica em um curso de graduação em matemática. 2019. Tese (Doutorado em Educação) Universidade Federal de Uberlândia, Uberlândia, 2019.

MINEIRO, R. M. Atividades para o estudo de superfícies quádricas mediadas por um modelo de representação tridimensional. 2011. Dissertação (Mestrado em Educação Matemática) - Universidade Bandeirante de São Paulo, São Paulo, 2011.

PAVANELLO, R. M. O abandono do ensino de geometria no Brasil: causas e consequências. Zetetiké, Campinas, v. 1, n. 1, p. 7-17, mar. 1993.

RICHIT, A. Projetos em geometria analítica usando software de geometria dinâmica: repensando a formação inicial docente em matemática. 2005. Dissertação (Mestrado em Educação Matemática) - Instituto de Geociências e Ciências Exatas, Universidade Estadual Paulista, Rio Claro, 2005.

SOUZA JUNIOR, A. J.; LOPES, E. M. C. Um mapeamento de pesquisas brasileiras sobre o trabalho educativo com tecnologias digitais de informação e comunicação no processo de ensinar e aprender Geometria Analítica. Vidya, Santa Maria, v. 37, n. 2, p. 479-497, jul-dez 2017.

VALE, I. Materiais Manipuláveis. Viana do Castelo: ESSE (Escola Superior de Educação), 2002.

VASCONCELOS, E. V.; ANDRADE, E. V.; CARDOSO, M. C. F.; SOUSA, M. G. P. Sólidos e superfícies: Construção de modelos concretos. Salvador: EDUFBA, 2010. 